ARTICLE

\title{
Optomechanical mass spectrometry
}

\author{
Marc Sansa (1) 1,7, Martial Defoort (10) 1,6,7, Ariel Brenac², Maxime Hermouet', Louise Banniard (1) 1, \\ Alexandre Fafin ${ }^{1}$, Marc Gely ${ }^{1}$, Christophe Masselon (10 ${ }^{3,4}$, Ivan Favero ${ }^{5}$, Guillaume Jourdan (1) ${ }^{1}$ \& \\ Sébastien Hentz (i) ${ }^{1 \times}$
}

Nanomechanical mass spectrometry has proven to be well suited for the analysis of high mass species such as viruses. Still, the use of one-dimensional devices such as vibrating beams forces a trade-off between analysis time and mass resolution. Complex readout schemes are also required to simultaneously monitor multiple resonance modes, which degrades resolution. These issues restrict nanomechanical MS to specific species. We demonstrate here single-particle mass spectrometry with nano-optomechanical resonators fabricated with a Very Large Scale Integration process. The unique motion sensitivity of optomechanics allows designs that are impervious to particle position, stiffness or shape, opening the way to the analysis of large aspect ratio biological objects of great significance such as viruses with a tail or fibrils. Compared to top-down beam resonators with electrical read-out and state-of-the-art mass resolution, we show a three-fold improvement in capture area with no resolution degradation, despite the use of a single resonance mode.

\footnotetext{
${ }^{1}$ Université Grenoble Alpes, CEA, LETI, 38000 Grenoble, France. ${ }^{2}$ Université Grenoble Alpes, CEA, CNRS, Grenoble INP, IRIG-Spintec, 38000 Grenoble, France. ${ }^{3}$ CEA, IRIG, Biologie à Grande Echelle, F-38054 Grenoble, France. ${ }^{4}$ Inserm, Unité 1038, F-38054 Grenoble, France. ${ }^{5}$ Matériaux et Phénomènes Quantiques, CNRS UMR 7162, Université de Paris, 75013 Paris, France. ${ }^{6}$ Present address: Université Grenoble Alpes, CNRS, Grenoble INP, TIMA, 38000 Grenoble, France. ${ }^{7}$ These authors contributed equally: Marc Sansa, Martial Defoort. ${ }^{凶}$ email: sebastien.hentz@cea.fr
} 
$\mathrm{M}$ ass spectrometry (MS) is an extraordinary versatile analytical technique ${ }^{1,2}$ that relies on ionization, electromagnetic fields to manipulate ions, and ensemble averaging of ions' mass-to-charge ratios to characterize species based on their specific molecular mass. Although MS was initially well suited for light objects such as molecules, it has been continuously making advances toward the characterization of ever larger supra-molecular assemblies ${ }^{3-5}$. Yet, no commercially available instrument can measure masses above a few MDa today.

An alternative approach consists in using nanomechanical resonators to determine the mass of individual particles accreting on their surface by measuring the induced frequency shift. Such nanomechanical mass sensing reached extremely low mass limits of detection ${ }^{6}$. As early systems relied on ionization and ion guides however, they suffered from the same limitations as conventional MS, which limited operation to the low MDa range together with prohibitively long analysis times and excessive sample requirements owing to the device's extremely low capture cross-section ${ }^{7}$. Eventually, the realization that nanomechanical resonators were ideally suited for analysis of masses in the MDa to GDa range opened the way for MS of species irrespective of charge ${ }^{8}$. Arrays of nanoresonators were demonstrated to circumvent the crosssection issue $e^{9,10}$ and $100 \mathrm{MDa}$ bacteriophage virus capsids were recently analyzed with a neutral nanomechanical MS system displaying high efficiency and resolution without the requirement for ionization ${ }^{11}$

The nanoresonators used in past mass experiments were all one-dimensional structures such as beams and cantilevers vibrating with flexural modes. With such devices, the frequency shift induced by a particle accreting on the resonator not only depends on mass, but it is also a function of landing position ${ }^{7,8}$. In some cases, particle stiffness ${ }^{12}$, size, and shape ${ }^{13}$ have to be accounted for. This has deleterious consequences: complex readout schemes must be used for simultaneous monitoring of multiple vibration modes; mass resolution dependence on landing position leads to a degradation of the average resolution by close to an order of magnitude compared with the optimum ${ }^{11}$, forcing a drastic compromise on capture area. Most importantly, the use of such one-dimensional resonators impedes the analysis of many crucial particles of biomedical interest having large aspect ratio, such as tailed viruses or fibrils, in particular when their length is similar to or exceeds the resonator's width.

In parallel with the developments employing electrically transduced nanoresonators, the field of cavity optomechanics has made critical advances ${ }^{14,15}$, approaching technological maturity. Its extreme displacement sensitivity and virtually unlimited bandwidth offers great promise for inertial ${ }^{16,17}$, chemical ${ }^{18}$, bio$\operatorname{logical}^{19}$, force ${ }^{20}$, mass ${ }^{21}$, and vibration ${ }^{22}$ sensing. This is particularly the case for nanomechanical MS, with the prospect of smaller and more sensitive resonators operating at higher frequencies and the possibility to design new device topologies enabled by the optomechanical transduction.

Here, we demonstrate single-particle mass spectrometry with optomechanical nanoresonators. These devices were fabricated with a unique Very Large Scale Integration process for optomechanics. Electrostatic actuation and released mechanical parts inherited from nano-electro-mechanical systems were used in combination with silicon photonics features such as on-chip light coupling and fiber packaging for vacuum and cryogenic operation. As opposed to electrical transductions used with prior devices, optomechanics is one of the very few transductions enabling new devices able to measure the mass of single particles in real time with a single vibration mode, featuring threefold larger capture area while maintaining similar mass resolution. This new device was designed to remain insensitive to particle landing position, stiffness, or aspect ratio, paving the way toward analyses of non-spherical particles, today very challenging for nanomechanical MS.

\section{Results}

Single-mode optomechanical nanoresonators. Our so-called "nano-ram" resonator consists in a sensing platform supported by four beams (Fig. 1a). A most interesting feature of this device lies in its in-plane rigid-body translation mode, in which the particle capture platform does not undergo deformation. The frequency shift induced by a particle on the platform thus remains independent from landing position (Fig. 1b), particle stiffness, shape, or aspect ratio (see Supplementary Fig. 1). In this scheme, singlemode operation significantly simplifies readout and data processing, mass sensitivity remains constant, and resolution optimal over the entire sensing area, whereas the larger capture area yields faster acquisition.

One of the main figures of merit of a resonator for mass sensing is the minimum detectable mass $\delta m_{\text {min }}$. It is given by

$$
\delta m_{\min }=2 M_{\text {res }}\left\langle\frac{\delta f}{f_{0 \text { min }}}\right\rangle
$$

where $M_{\text {res }}$ is the total mass of the platform (see Supplementary Fig. 2) and $\frac{\delta f}{f_{0} \min }$ its frequency stability. Both factors should remain as small as possible to improve mass resolution. In order to maintain a large capture area while minimizing its total mass, the platform and its supports were thinned down to $60 \mathrm{~nm}$. The frequency stability is ultimately limited by intrinsic fluctuations of the resonance frequency in the mechanical domain ${ }^{23}$. Reaching this limit experimentally requires large signal-to-noise ratio (SNR), which is maximized when the resonator is driven up to the onset of mechanical non-linearity and when its thermomechanical noise dominates. Although this is achievable for bulky devices in in-plane rigid-body motion with electrical transduction $^{24,25}$, it becomes extremely challenging for ordersof-magnitude lighter, smaller, and higher-frequency resonators with a few 10's nm thickness such as ours. It is nevertheless within reach of optomechanical transduction, thanks to the remarkable displacement sensitivity. Conversely, electrostatic actuation was needed to drive the resonator to high amplitudes with low voltage, eventually up to the onset of non-linearity. These dual requirements called for integration of both optical and electrical inputs/outputs in the same fabrication process. A scanning electron microscope image shows all elements of the device (Fig. 1c), fabricated in an industrial-grade clean room; a schematic cross-section is shown Fig. 1d (see Methods for a detailed description of the fabrication process).

The nanoresonator's motion detunes the optical ring cavity and, in the bad cavity limit where we operate ${ }^{15}$, modulates the output optical power at the mechanical resonance frequency (Fig. 2a, inset) with an amplitude $\Delta P$ per unit displacement: $\Delta P \propto$ $Q_{\text {opt }} C_{\mathrm{r}} g_{\mathrm{om}}{ }^{16}$. The loaded optical quality factor $Q_{\mathrm{opt}}$ depends on both intrinsic and extrinsic losses, which are of equal value at the so-called critical coupling, when the contrast $C_{\mathrm{r}}$ is equal to $1^{26}$. We designed a single-mode, $10 \mu \mathrm{m}$ radius ring resonator (Supplementary note 1 ) coupled to a close-by waveguide (200 $\mathrm{nm}$ gap). This conservative design leads to operation in an undercoupling regime, with $C_{\mathrm{r}} \sim 0.23$ and typical loaded quality factors in the order of $5 \times 10^{4}$ (Fig. $2 \mathrm{~b}$ and Supplementary Fig. 3). The dominant loss mechanism was attributed to scattering owing to interaction with the ring anchoring spokes ${ }^{27}$, even though these were tapered at their extremities, down to $\sim 100 \mathrm{~nm}$. Setting the laser wavelength close to the point of maximum slope of the optical resonance, and demodulating the transmitted light power at a frequency close to the nanoresonator's resonance frequency, we measured the thermomechanical noise of the mechanical 
a

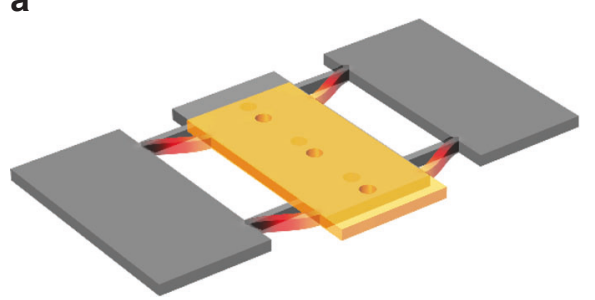

C

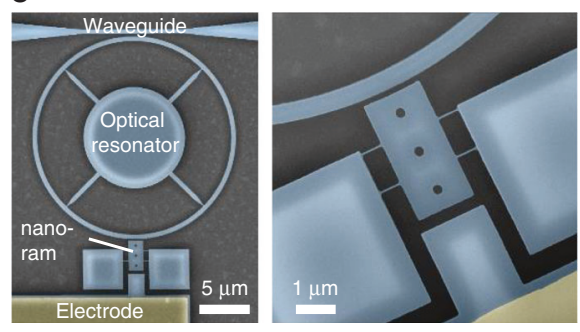

b

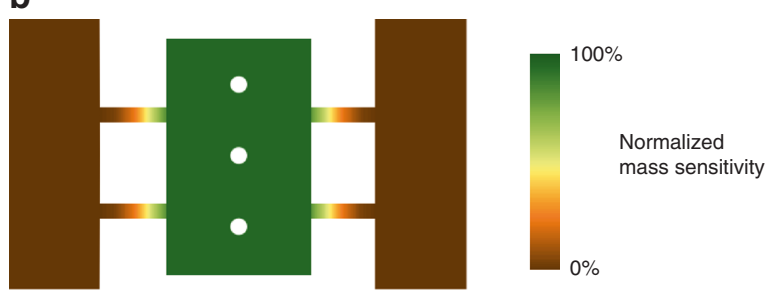

d

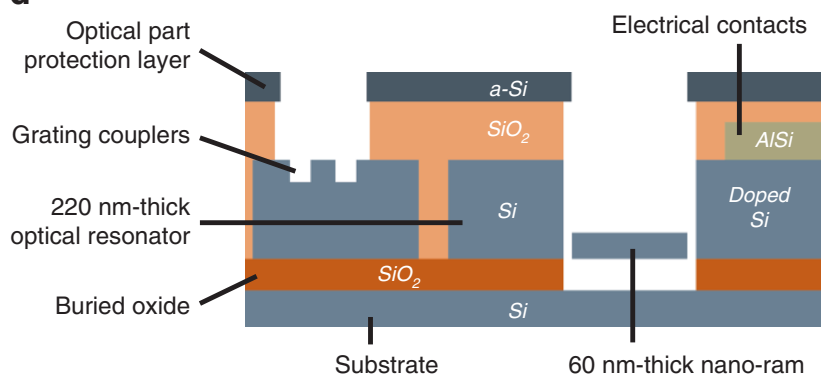

Fig. 1 Single-mode optomechanical resonator for mass spectrometry. a In-plane rigid-body vibration mode of interest of the nanomechanical resonator (see Supplementary Fig. 2 for the other modes). b Finite-element color map of normalized frequency sensitivity to added point mass, showing that the frequency shift due to particle adsorption does not depend on particle position on the platform. $\mathbf{c}$ False-colored scanning electron microscope images of the device, general view (left), and zoom-in on the nano-ram (right). The platform is $1.5 \mu \mathrm{m}$ wide and $3 \mu \mathrm{m}$ long, with $80 \times 500 \mathrm{~nm}$ support beams. The optical ring diameter is $20 \mu \mathrm{m}$, and the optical ring-to-platform gap is $100 \mathrm{~nm}$. Close to $1.55 \mu \mathrm{m}$ wavelength light is coupled in and out of the ring by optical waveguides through a $200 \mathrm{~nm}$ gap. Electrostatic actuation is performed with a side-gate $250 \mathrm{~nm}$ away from the nanoresonator. d Cross-section showing the different components of the device. The silicon-on-insulator (SOI) top layer is $220 \mathrm{~nm}$ thick, partially etched to realize the optical grating couplers. The nanoresonator is etched down to $60 \mathrm{~nm}$. The crystalline Si layer is highly doped locally for low metal-to-silicon contact resistance. A $200 \mathrm{~nm}$ amorphous silicon layer is deposited above a planarized silicon oxide layer for protection and etched open above the grating couplers and the nano-ram.

resonator (Fig. 2c, inset). This allowed the determination of the optomechanical coupling factor $g_{\mathrm{om}}{ }^{28}$, equal to $0.4 \mathrm{GHz} \mathrm{nm}^{-1}$ (Supplementary note 1). Light was coupled on-chip via grating couplers, designed so their maximum transmission was close to $1550 \mathrm{~nm}$ wavelength ${ }^{29}$ (Fig. 3b).

Figure 2a shows the measurement set-up, featuring optomechanical detection and electrical actuation. The nanoresonator's frequency responses were measured with increasing drive voltage (Fig. 2c). As actuation and motion sensing were not performed in the same physical domain, they were very well decoupled, ensuring a large signal-to-background ratio (Supplementary Fig. 5). The frequency stability $\frac{\delta f}{f_{0} \text { min }}$ was measured by tracking the resonance frequency of the resonator in closed loop using a phase-locked-loop (PLL, Supplementary Fig. 6a), and plotting the Allan deviation ${ }^{30}$ for different drive voltages. Each plot was compared with open loop operation to make sure that the frequency stability was not affected by the PLL corrector (Supplementary Fig. 6b). At low integration times and low drive voltages, i.e., in the regime where additive white noise dominates, the Allan deviation scaled like $\tau^{-1 / 2}$ and improved linearly with drive amplitude, as expected ${ }^{23}$. Above some voltage threshold, the stability stopped improving and settled into a limit in the ppm range. As this threshold was well below the onset of non-linearity, operation in the non-linear regime was not required ${ }^{31}$. This behavior was consistent with the presence of mechanical resonance frequency fluctuations ${ }^{23}$. Despite the modest colocalization of mechanical motion and optical mode (owing to the small thickness and lateral dimensions of the mechanical resonator), and despite small displacement and high frequency, the optomechanical transduction proved able to resolve thermomechanical noise (Fig. 2c) and to reach the ultimate stability limit allowed by the mechanical device (Fig. 2d). With a $1 \mathrm{ppm}$ frequency stability and a $608 \mathrm{fg}$ mass, Eq. 1 yields a $0.7 \mathrm{MDa}$ $(1.2 \mathrm{ag})$ minimum detectable mass. This was similar to the average mass resolution obtained with previously reported piezoresistive beam resonators $\left(0.5 \mathrm{MDa}^{11}\right)$, albeit with a threefold increased capture area and a resonator topology immune to particle's shape, stiffness and landing position.

Optomechanical mass spectrometry. In order to maintain a stable optical transduction during mass sensing experiments, the optical elements were protected with a $\sim 200 \mathrm{~nm}$-thick amorphous silicon layer (see cross-section Fig. 1d) etched open only on top of the resonator's sensing platform (Fig. 3d). This protective layer avoided degradation of both optical transmission and optomechanical coupling as well detuning of the optical cavity owing to accretion of particles on the optical waveguide and resonator (Supplementary Fig. 7). The alignment uncertainty between lithography levels was key in this context and sub-100 nm alignment was obtained (see Methods).

An additional challenge for routine mass sensing experiments was efficient light coupling on-chip within a spatially constrained particle-generating system under vacuum and at low temperature. Prior optomechanical works used tapered optical fibers directly coupled to the cavity ${ }^{32}$ or microlensed fibers coupled to tapered waveguides $^{33}$ but both techniques require piezoelectric stages and proper vibration control. Light can also be focused with an objective lens onto grating couplers from outside the system ${ }^{18}$ but this requires a device in immediate proximity and parallel to a window. In this work, we used fiber-transposer chips (Teem Photonics) that were UV-glued onto the grating couplers (Fig. 3b, c and Supplementary Fig. 8). This silicon photonics-derived technique allowed small footprint, high density (grating couplers are $\sim 500 \mu \mathrm{m}$ away from the device) and high transmission. Standard vacuum feedthroughs could thus be used to route both electrical and optical signals in and out of the system (Fig. 3a).

Mass spectrometry experiments were performed in a fourvacuum chambers system described in more detail elsewhere ${ }^{34}$, 


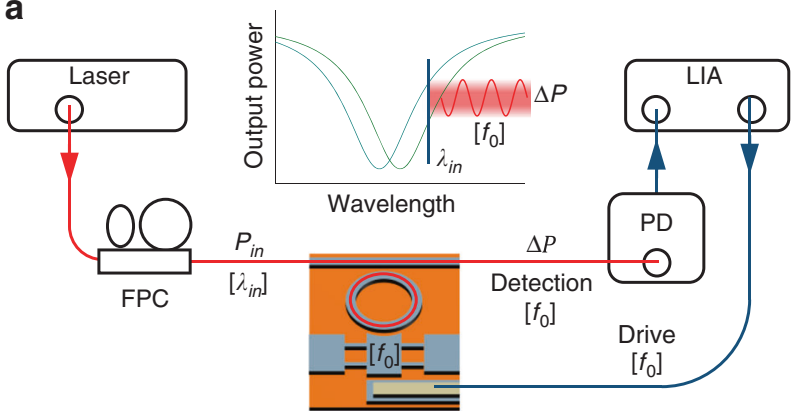

C

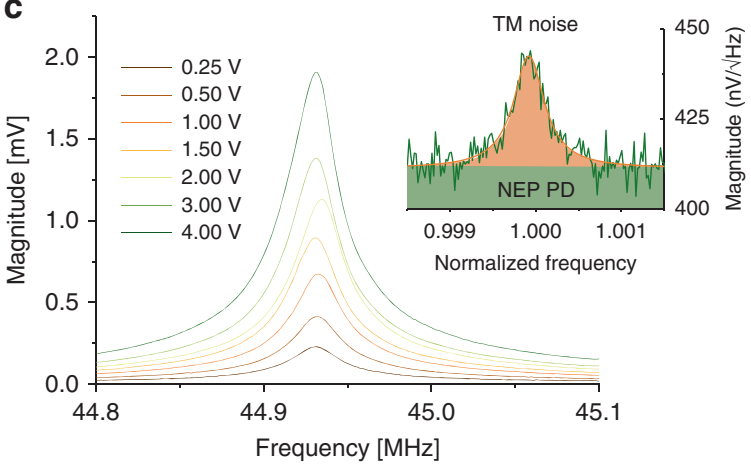

b

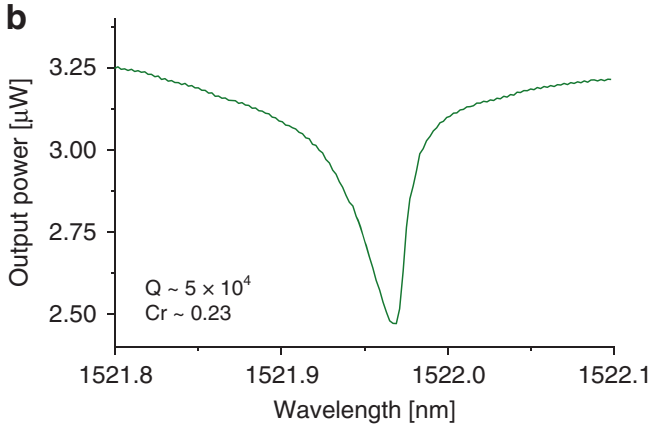

d

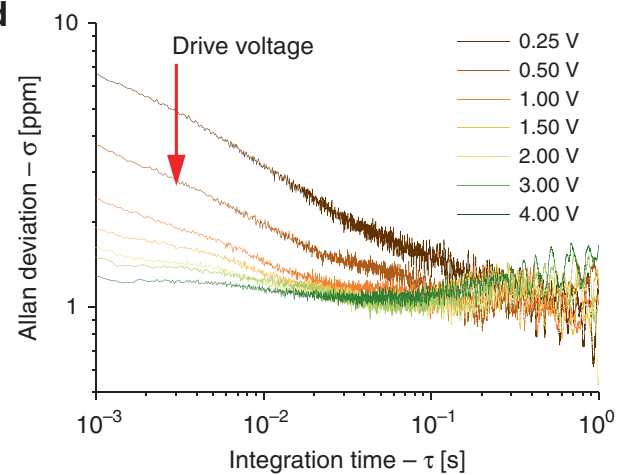

Fig. 2 Optical, optomechanical, and frequency stability measurements of the nanoresonator at $\sim \mathbf{1 0}^{-\mathbf{5}}$ Torr and $\mathbf{7 7} \mathbf{~ K}$. a Optomechanical readout scheme. FPC stands for fiber-polarization controller, PD for photodetector, and LIA for lock-in amplifier. Red and dark blue lines are optical and electrical signals, respectively. The nanomechanical resonator vibrating at frequency $f_{0}$ modulates the resonance wavelength of the optical cavity and consequently the light output power at $f_{0}$. b Narrow-band optical transmission spectrum around one resonance of the ring resonator measured with $0.5 \mathrm{~mW}$ output laser power. A slight thermo-optic behavior is observed. The loaded optical quality factor is $\sim 5 \times 10^{4}$, and the contrast is 0.23 . c Optomechanical spectra of the first inplane mode close to $44.9 \mathrm{MHz}$ with increasing drive voltages. The optical input power is set to $10 \mathrm{~mW}$ (Supplementary Fig. 4). The mechanical quality factor is 1700 . Inset: thermomechanical noise of the resonator. The noise floor (green) is set by the photodetector's amplifier input noise, equivalent to a displacement resolution of $2.8 \times 10^{-14} \mathrm{~m} \mathrm{~Hz}^{-\frac{1}{2}}$. d Allan deviation of the resonator, using a PLL. When increasing the drive voltage, the stability falls into a $1 \mathrm{ppm}$ limit over the whole integration time range, which is consistent with the presence of mechanical resonance frequency fluctuations.

that comprised a sputtering source and an in-line time-of-flight mass spectrometer (TOF-MS). Metallic nanoclusters beams with tunable size and deposition rate were generated using sputtering gas-aggregation technique. Nanoclusters were expelled through a differential pumping stage (Fig. 3a) into a vacuum deposition chamber $\left(10^{-5}\right.$ Torr $)$ containing our optomechanical device mounted onto a translational stage that could be inserted into the cluster beam. Upon retraction of this stage, the particle flux entered the in-line TOF mass spectrometer, where the mass-tocharge distribution of charged particles could be determined. The configuration of the deposition chamber thus allowed TOF-MS and optomechanical MS measurements sequentially on the same cluster population.

We selected tantalum as analyte as it is both dense $\left(16.6 \mathrm{~g} \mathrm{~cm}^{-3}\right)$ and readily condenses into large clusters. Detecting such large clusters with the TOF mass spectrometer constituted a first challenge. We were able to detect clusters up to $\sim 7 \mathrm{MDa}$ by TOFMS, i.e., close to twofold heavier compared with previous experiments ${ }^{8,9}$. This was achieved by an increase in the ion acceleration voltage (from 3 to $3.6 \mathrm{kV}$ ), as the ion detector efficiency is proportional to the square of this parameter. Monitoring the nanoresonator's frequency was achieved with a PLL with a response time of $\sim 10 \mathrm{~ms}$. An example of mechanical frequency trace in closed-loop operation acquired during exposure to cluster beam is shown Fig. 4a. Frequency jumps (see inset) were converted into mass with $\Delta m=2 M_{\text {res }} \Delta f / f_{0}$. In contrast with prior devices, landing position, stiffness, or aspect ratio did not have to be accounted for (see Supplementary Fig. 1) and direct access to the mass information was possible. Tuning of the nanocluster source parameters yielded particle adsorption event rates on the order of four events per second, making the landing of several particles within the PLL response time very unlikely. Notably, we observed a threefold improvement in event rate compared with one-dimensional vibrating beams with a similar particle flux ${ }^{8,9}$, which was in agreement with the capture surface ratio of the resonator used. Particle landing events were combined into histograms with bin widths ranging from 0.5 to $1 \mathrm{MDa}$. Mass spectra of four different nanocluster populations $(2.8,3.9,5.7$, and 7.7 MDa) acquired by both optomechanical MS and TOF-MS are displayed in Fig. 4. Although optomechanical MS directly provided the cluster mass distribution, TOF-MS yielded mass-to-charge ratio distributions. Although the vast majority of clusters carried only one charge, the presence of multiple charge states and the low TOF SNR could make spectra interpretation complex (Supplementary Fig. 12). Each optomechanical MS spectrum was acquired in only $5 \mathrm{~min}$, and comprised $\sim 1000$ events. Both techniques provided comparable mean mass estimates for low mass populations (2.76 vs $2.83 \mathrm{MDa}$ and $3.85 \mathrm{vs.} 3.82 \mathrm{MDa}$ for optomechanical MS and TOF-MS, respectively). Nevertheless, the optomechanical spectra consistently displayed a larger proportion of higher mass particles than TOF-MS and this was even more so for high-mass particles (5.67 and 7.68 MDa). This could be attributed to the fact that measurements were performed at the extreme mass range achievable for our TOF-MS: detection efficiency being roughly inversely proportional to ion mass, a fast decay of the detected signal with particle mass was expected, causing a distortion of the spectrum. This in turn degraded the detector's SNR, making baseline subtraction more difficult, and may have resulted in a 
a

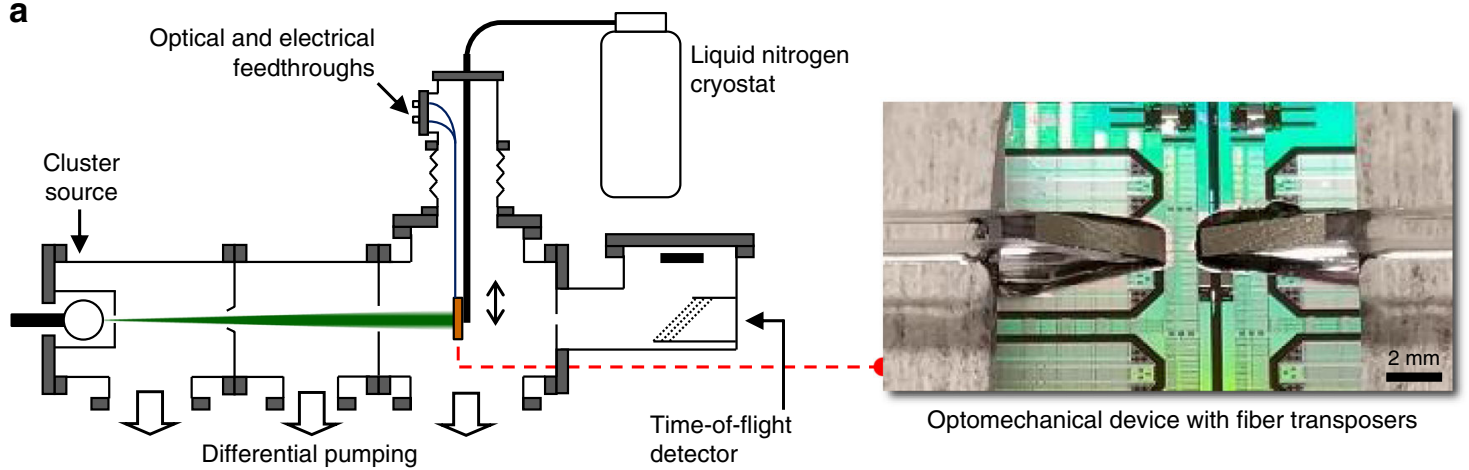

b

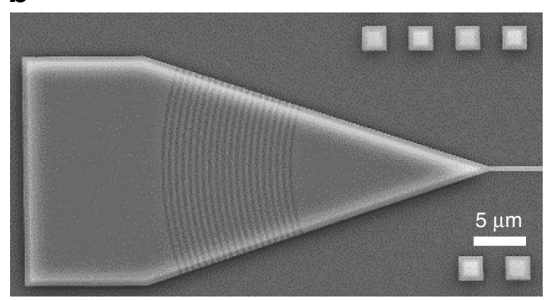

C

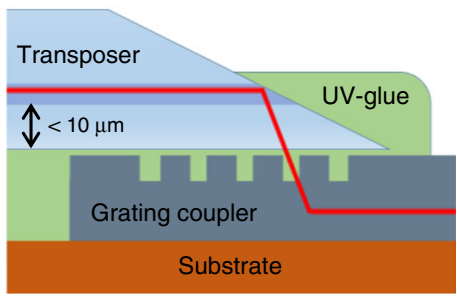

d

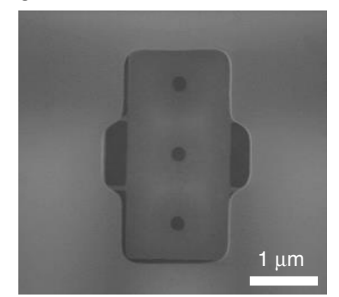

Fig. 3 Vacuum system used for mass deposition and optically packaged optomechanical device. a The set-up consists in a sputtering source capable of generating metallic clusters of controlled size and mass, and a time-of-flight spectrometer. Vacuum feedthroughs carry electrical and optical signals in and out of the system. The optomechanical device is placed on a retractable sample holder at the end of a cryostat (liquid nitrogen). By moving the sample holder in and out of the cluster beam, a given cluster population can be measured with the nanoresonator and TOF detector sequentially. $\mathbf{b}$ Light is coupled in and out of the optomechanical chips using grating couplers with a pitch of $0.6 \mu \mathrm{m}$ and a width of $0.3 \mu \mathrm{m}$, designed for maximum transmission close to a $1550 \mathrm{~nm}$ wavelength and an input angle of $10^{\circ 29}$. c Quasi in-plane optical packaging by waveguide-to-fiber-transposer chips (measuring $1 \times 2 \times 20 \mathrm{~mm}$ ), aligned and glued to the grating couplers. The electrical inputs/outputs are obtained by standard wire-bonding on metallic pads (see also Supplementary Fig. 9). d SEM image of the amorphous silicon protection layer covering all optical and electrical elements so that mass deposition only occurs on the sensing platform.

a

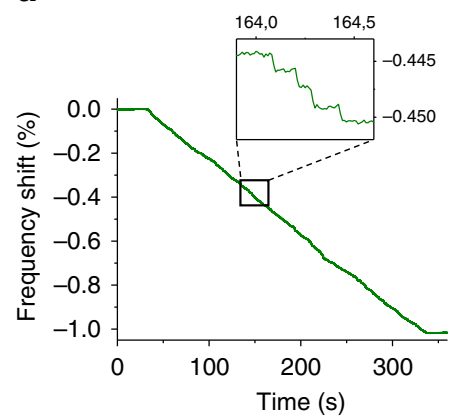

b

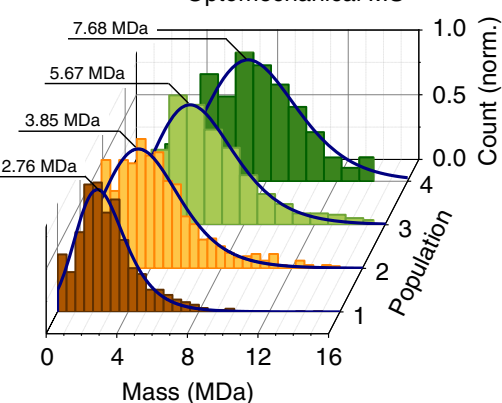

C

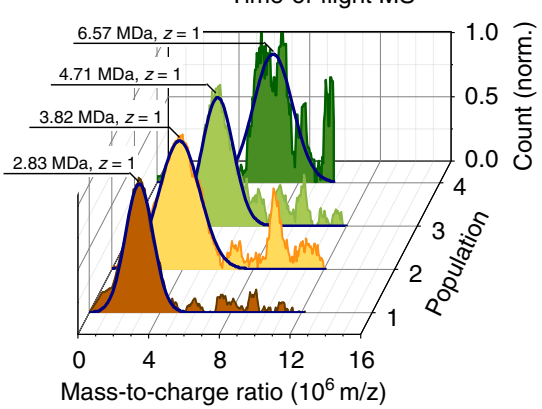

Fig. 4 Single-particle optomechanical mass spectrometry of tantalum clusters. a Frequency trace of the optomechanical resonator for the light green (5.7 MDa) cluster population deposition. For this particular measurement, 1140 events were recorded in $\sim 5$ min, for a total deposited mass close to $1 \%$ of the mass of the resonator. The sampling time is here $50 \mu \mathrm{s}$, the PLL bandwidth is $10 \mathrm{~ms}$ and the plotted frequency trace is averaged to a $10 \mathrm{~ms}$ integration time. Spectra are plotted from detected frequency jump heights (Methods). Inset: detail showing several frequency jumps from individual cluster depositions. b Normalized nanoresonator's and c TOF mass spectra for four different tantalum cluster populations fitted with a log-normal function (dark blue lines) with mean masses ranging from 2.7 to $7.7 \mathrm{MDa}$ (for optomechanical MS), equivalent to particle diameters from 8 to $11.3 \mathrm{~nm}$. The number of events in the nanoresonator's spectra range from 534 to 1384, for measurement times up to 300 sec in each case.

high-mass cutoff, explaining the underestimation of the average mass and of the standard deviation. This was prominently observed with the population with the largest mass, for which obtaining a spectrum with TOF-MS proved very challenging and required long integration times. Conversely, the nanoresonator limit of detection remaining constant with mass, its resolving power (ratio of analyzed mass-to mass resolution) increased with mass; and high-mass events were detected with better relative precision. This suggests that the high-mass tails of the optomechanical spectra were very likely representative of the cluster population, whereas TOF-MS "missed" these clusters (see Methods and Supplementary Figs. 10 and 11). This most likely explains the discrepancy in mean mass for the two heavier cluster populations. On the other hand, the low mass events (typically below $3 \mathrm{MDa}$ ) were performed close to the resonator's limit of detection ( $0.7 \mathrm{MDa})$. Measurement of the resonator's frequency noise was required to correctly interpret the associated spectra in this range (see Methods). In total, our optomechanical resonator 
accumulated close to $10 \%$ of its initial mass in less than an hour of deposition without measurable performance degradation (Supplementary Fig. 13). The optical response remained constant during the whole process, without noticeable changes in quality factor, transmission, or resonance wavelength detuning (Supplementary Fig. 7).

\section{Discussion}

Nanoresonators used in nanomechanical MS experiments to date were one-dimensional structures such as beams and cantilevers vibrating with flexural modes. With such structures, the frequency shift induced by accretion of a particle also depends on landing position: measuring mass requires determining particle position on the beam. This is performed using a complex readout scheme designed to simultaneously monitor the frequency of at least two resonance modes ${ }^{7,8}$. In addition, the achievable mass resolution also depends on the landing position, and only close to half the total resonator's area is effectively used in the measurements $^{7-9}$. This in turns leads to reduced capture cross-section, longer analysis times and five to ten times larger average mass uncertainty compared with the best performance attainable on the beam ${ }^{11}$. Moreover, measuring stiffness, size, or shape may be required to correctly determine mass, which means monitoring an even higher number of modes ${ }^{12,13}$. At last, measuring the mass of elongated objects, if possible at all, would produce significant mass errors, in particular when their length is larger than the beam's lateral dimension.

The present report constitutes a significant departure from previous work as we successfully performed single-particle mass spectrometry with a resonator that requires monitoring a single vibration mode and a simple and efficient readout scheme. This resonator is by design insensitive to landing position, particle stiffness, size, shape, or aspect ratio. It embeds a platform that vibrates in-plane with a rigid-body motion, providing large capture cross-section. The sensing platform was thinned down in order to obtain low resonator mass and high frequency-to-mass sensitivity. Obtaining excellent mass resolution requires large SNR, large dynamic range and high displacement sensitivity. This is extremely difficult to achieve with electrical transductions for such thin, in-plane devices and we used here on-chip cavity-based optomechanical resonators for this purpose. They were fabricated with a Very Large Scale Integration fabrication process for optomechanics, on $200 \mathrm{~mm}$ wafers. Using this device, we have performed MS analysis of tantalum nanoclusters ranging from 2.8 to $7.7 \mathrm{MDa}$ in $<5$ minutes, and demonstrated an excellent stability of the sensor during these depositions.

This demonstration of on-chip optomechanics as a superior alternative to electromechanical resonator for high-resolution single-particle mass spectrometry paves the way to highthroughput MS analysis of synthetic and natural nanoparticles with any possible geometry. For this purpose, our photonicsderived fabrication process is easily amenable to multiplexing of a large number of resonators using standard wavelength-division multiplexing ${ }^{35}$ and packaging techniques. In addition, although our resonators were etched down to $60 \mathrm{~nm}$, this thickness could be reasonably reduced to $\sim 10 \mathrm{~nm}$ in the future. Mass resolution below $100 \mathrm{kDa}$ could be reached with such lighter resonators and much stiffer anchors, yielding resonance frequency above $500 \mathrm{MHz}$, still in reach of optomechanical readout ${ }^{36}$. Such devices would open new applications in structural biology and nanocharacterization by achieving the performance required for the analysis of non-spherical objects that are challenging for current nanomechanical MS, such as amyloid fibrils involved in neurodegenerative diseases, or tailed viruses used for phagotherapy, a promising alternative to standard antibiotherapy.

\section{Methods}

Fabrication process. The sensors were fabricated with a Very Large Scale Integration fabrication process in an industrial-grade clean room. Starting from $200 \mathrm{~mm}$ Silicon-on-Insulator wafers with $220 \mathrm{~nm}$ thick silicon top layer, a first lithography and partial etching $(70 \mathrm{~nm})$ of the grating couplers are performed, followed by a second similar step to reduce the thickness of the nano-ram (the final thickness is measured by both Focused Ion Beam and spectroscopic ellipsometry). Next, the waveguides, optical cavities and mechanical resonators are patterned by dry etching. We developed a specific lithography step by variable shape beam for this step. This technique allows both smooth pattern walls and low optical losses, and full electron beam resist insulation over the whole wafer in a reasonable amount of time (a few hours). Silicon is locally highly doped (boron, $5 \times 10^{19} \mathrm{at} \mathrm{cm}^{-3}$ ) for electrical actuation while preserving good optical properties elsewhere. A metal layer is deposited and patterned for electrical contacts. Then a sacrificial layer (silicon oxide) is deposited everywhere and planarized, followed by a deposition of a $\sim 200 \mathrm{~nm}$-thick amorphous silicon layer, which is etched open only on top of the resonator's sensing platform. A good alignment (below $100 \mathrm{~nm}$ ) is critical for this step, as the aperture has to be located precisely on top of the platform. Finally the nanoresonator is released by vapor HF etching. Figure $1 \mathrm{~d}$ shows a simplified cross-section of the device.

Frequency data processing for spectrum plot. The frequency time traces are averaged with an averaging time depending on the event rate $(10 \mathrm{~ms}$ for the three first mass spectra and $20 \mathrm{~ms}$ for the highest mass spectrum). This averaging time is a trade-off between noise averaging and single-particle detection. The resulting traces are scanned for abrupt changes to detect particle landing events. Because frequency traces are sampled, the jumps may spread over several sampling times. Therefore, their heights are quantified by measuring frequency before and after these abrupt changes and masses of the particles are inferred from those spread jumps. Masses are then binned to plot the histograms. In addition, frequency noise may produce false positives when dealing with masses close to the nanoresonator's resolution. We perform a statistical subtraction of these events by measuring the nanoresonator's resonance frequency for $30 \mathrm{~s}$ before and after each deposition run. "Mass" histograms are built with these sets of frequency noise and their height is subsequently scaled as a function of the deposition run duration. Each bin value of the noise histograms is then subtracted from the deposited mass histogram.

\section{Data availability}

The data that supports the findings of this study are available from the corresponding author upon reasonable request.

Received: 24 March 2020; Accepted: 1 July 2020;

Published online: 29 July 2020

\section{References}

1. Heberer, T. Occurrence, fate, and removal of pharmaceutical residues in the aquatic environment: a review of recent research data. Toxicol. Lett. 131, 5-17 (2002).

2. Robinson, C. V., Sali, A. \& Baumeister, W. The molecular sociology of the cell. Nature 450, 973-982 (2007)

3. Snijder, J., Rose, R. J., Veesler, D., Johnson, J. E. \& Heck, A. J. R. Studying 18 $\mathrm{MDa}$ virus assemblies with native mass spectrometry. Angew. Chem. - Int. Ed. 52, 4020-4023 (2013).

4. Doussineau, T. et al. Mass determination of entire amyloid fibrils by using mass spectrometry. Angew. Chem. Int. Ed. 55, 2340-2344 (2016).

5. Keifer, D. Z., Motwani, T., Teschke, C. M. \& Jarrold, M. F. Measurement of the accurate mass of a $50 \mathrm{MDa}$ infectious virus. Rapid Commun. Mass Spectrom. 30, 1957-1962 (2016).

6. Chaste, J. et al. A nanomechanical mass sensor with yoctogram resolution. Nat. Nanotechnol. 7, 301-304 (2012).

7. Hanay, M. S. et al. Single-protein nanomechanical mass spectrometry in real time. Nat. Nanotechnol. 7, 602-608 (2012).

8. Sage, E. et al. Neutral particle mass spectrometry with nanomechanical systems. Nat. Commun. 6, 6482 (2015).

9. Sage, E. et al. Single-particle mass spectrometry with arrays of frequencyaddressed nanomechanical resonators. Nat. Commun. 9, 3283 (2018).

10. Stassi, S. et al. Large-scale parallelization of nanomechanical mass spectrometry with weakly-coupled resonators. Nat. Commun. 10, 3647 (2019).

11. Dominguez-Medina, S. et al. Neutral mass spectrometry of virus capsids above 100 megadaltons with nanomechanical resonators. Science 362, 918-922 (2018).

12. Malvar, O. et al. Mass and stiffness spectrometry of nanoparticles and whole intact bacteria by multimode nanomechanical resonators. Nat. Commun. 7, 13452 (2016).

13. Sader, J. E., Hanay, M. S., Neumann, A. P. \& Roukes, M. L. Mass spectrometry using nanomechanical systems: beyond the point-mass approximation. Nano Lett. 18, 1608-1614 (2018). 
14. Favero, I. \& Karrai, K. Optomechanics of deformable optical cavities. Nat. Photonics 3, 201-205 (2009).

15. Aspelmeyer, M., Kippenberg, T. J. \& Marquardt, F. Cavity optomechanics. Rev. Mod. Phys. 86, 1391 (2014)

16. Krause, A. G., Winger, M., Blasius, T. D., Lin, Q. \& Painter, O. A highresolution microchip optomechanical accelerometer. Nat. Photonics $\mathbf{6}$, 768-772 (2012).

17. Dong, B., Cai, H., Tsai, J. M., Kwong, D. L. \& Liu, A. Q. An on-chip optomechanical accelerometer. In 2013 IEEE 26th International Conference on Micro Electro Mechanical Systems (MEMS) 641-644 (2013).

18. Venkatasubramanian, A. et al. Nano-optomechanical systems for gas chromatography. Nano Lett. 16, 6975-6981 (2016).

19. Yu, W., Jiang, W. C., Lin, Q. \& Lu, T. Cavity optomechanical spring sensing of single molecules. Nat. Commun. 7, 1-9 (2016).

20. Etienne Allain, P. et al. Optomechanical resonating probe for very high frequency sensing of atomic forces. Nanoscale 12, 2939-2935 (2020).

21. Liu, F., Alaie, S., Leseman, Z. C. \& Hossein-Zadeh, M. Sub-pg mass sensing and measurement with an optomechanical oscillator. Opt. Express 21, 19555 (2013).

22. Gil-Santos, E. et al. Optomechanical detection of vibration modes of a single bacterium. Nat. Nanotechnol. 15, 467-474 (2020).

23. Sansa, M. et al. Frequency fluctuations in silicon nanoresonators. Nat. Nanotechnol. 11, 552-559 (2016).

24. Rahafrooz, A. \& Pourkamali, S. Fabrication and characterization of thermally actuated micromechanical resonators for airborne particle mass sensing: I. Resonator design and modeling. J. Micromech. Microeng. 20, 125018 (2010).

25. Perelló-Roig, R., Verd, J., Barceló, J., Bota, S. \& Segura, J. A 0.35- $\mu$ m CMOSMEMS oscillator for high-resolution distributed mass detection. Micromachines 9, 484 (2018).

26. Ding, L. et al. Gallium Arsenide Disk Optomechanical Resonators. in Handbook of Optical Microcavities 526 (Pan Stanford, 2014).

27. Schwab, L. et al. Comprehensive optical losses investigation of VLSI Silicon optomechanical ring resonator sensors. In 2018 IEEE International Electron Devices Meeting (IEDM) San Francisco, CA, pp. 4.7.1-4.7.4 (2018).

28. Gorodetksy, M. L., Schliesser, A., Anetsberger, G., Deleglise, S. \& Kippenberg, T. J. Determination of the vacuum optomechanical coupling rate using frequency noise calibration. Opt. Express 18, 23236-23246 (2010).

29. Bernabé, S. et al. Chip-to-chip optical interconnections between stacked selfaligned SOI photonic chips. Opt. Express 20, 7886-7894 (2012).

30. Allan, D. W. Time and frequency (time-domain) characterization, estimation, and prediction of precision clocks and oscillators. IEEE Trans. Ultrason. Ferroelectr. Freq. Control 34, 647-654 (1987).

31. Yuksel, M. et al. Nonlinear nanomechanical mass spectrometry at the singlenanoparticle level. Nano Lett. 19, 3583-3589 (2019).

32. MacDonald, A. J. R. et al. Optical microscope and tapered fiber coupling apparatus for a dilution refrigerator. Rev. Sci. Instrum. 86, 013107 (2015)

33. Gil Santos, E. et al. Light-mediated cascaded locking of multiple nanooptomechanical oscillators. Phys. Rev. Lett. 118, 063605 (2017).

34. Morel, R., Brenac, A., Bayle-Guillemaud, P., Portemont, C. \& Rizza, F. L. Growth and properties of cobalt clusters made by sputtering gas-aggregation. Eur. Phys. J. 24, 287-290 (2003).

35. Sauer, V. T. K., Diao, Z., Freeman, M. R. \& Hiebert, W. K. Wavelengthdivision multiplexing of nano-optomechanical doubly clamped beam systems. Opt. Lett. 40, 1948 (2015).
36. Ding, L. et al. High frequency GaAs nano-optomechanical disk resonator. Phys. Rev. Lett. 105, 263903 (2010).

\section{Acknowledgements}

S.H. acknowledges support from the European Union through the ERC Enlightened project (616251), I.F. through the ERC Nomli project (N770933) and M.S. through the Marie-Curie Eurotalents incoming fellowship. We thank Florent Gardillou (Teem Photonics) for his help with the optical packaging.

\section{Author contributions}

M.S., M.D., M.H., L.B., A.F., I.F., G.J., and S.H. designed the optomechanical devices M.S. and M.D. performed the optical and optomechanical tests. M.S., M.D., and A.B. performed the mass spectrometry experiments. M.S., M.D., A.B., C.M., I.F., G.J., and S.H. interpreted the data. M.G. supervised the device fabrication. All authors contributed to the writing of the manuscript.

\section{Competing interests}

S.H. and M.S. are co-inventors of patents No. EP3509214A1, US20190204205A1, and CN109994364A

\section{Additional information}

Supplementary information is available for this paper at https://doi.org/10.1038/s41467020-17592-9.

Correspondence and requests for materials should be addressed to S.H.

Peer review information Nature Communications thanks Wayne Hiebert, Carlo Ricciardi and the other, anonymous, reviewer(s) for their contribution to the peer review of this work.

Reprints and permission information is available at http://www.nature.com/reprints

Publisher's note Springer Nature remains neutral with regard to jurisdictional claims in published maps and institutional affiliations.

Open Access This article is licensed under a Creative Commons Attribution 4.0 International License, which permits use, sharing, adaptation, distribution and reproduction in any medium or format, as long as you give appropriate credit to the original author(s) and the source, provide a link to the Creative Commons license, and indicate if changes were made. The images or other third party material in this article are included in the article's Creative Commons license, unless indicated otherwise in a credit line to the material. If material is not included in the article's Creative Commons license and your intended use is not permitted by statutory regulation or exceeds the permitted use, you will need to obtain permission directly from the copyright holder. To view a copy of this license, visit http://creativecommons.org/ licenses/by/4.0/.

(C) The Author(s) 2020 